\title{
Correction of Spondylolisthesis by Lateral Lumbar Interbody Fusion Compared with Transforaminal Lumbar Interbody Fusion at L4-5
}

\author{
Myeong Jin Ko, Seung Won Park, Young Baeg Kim \\ Department of Neurosurgery, Chung-Ang University Hospital, Seoul, Korea
}

Objective : In an aging society, the number of patients with symptomatic degenerative spondylolisthesis (DS) is increasing and there is an emerging need for fusion surgery. However, few studies have compared transforaminal lumbar interbody fusion (TLIF) and lateral lumbar interbody fusion (LLIF) for the treatment of patients with DS. The purpose of this study was to investigate the clinical and radiological outcomes between TLIF and LLIF in DS.

Methods : We enrolled patients with symptomatic DS at L4-5 who underwent TLIF with open pedicle screw fixation (TLIF group, $\mathrm{n}=41$ ) or minimally invasive LLIF with percutaneous pedicle screw fixation (LLIF group, $\mathrm{n}=39$ ) and were followed-up for more than one year. Clinical (visual analog scale and Oswestry disability index) and radiological outcomes (spondylolisthesis rate, segmental sagittal angle [SSA], mean disc height [MDH], intervertebral foramen height [FH], cage subsidence, and fusion rate) were assessed. And we assessed the changes in radiological parameters between the postoperative and the last follow-up periods.

Results : Preoperative radiological parameters were not significantly different between the two groups. LLIF was significantly superior to TLIF in immediate postoperative radiological results, including reduction of spondylolisthesis rate (3.8\% and $7.2 \%)$, increase in $\mathrm{MDH}(13.9 \mathrm{~mm}$ and $10.3 \mathrm{~mm})$ and $\mathrm{FH}(21.9 \mathrm{~mm}$ and $19.4 \mathrm{~mm})$, and correction of SSA $\left(18.9^{\circ}\right.$ and $\left.15.6^{\circ}\right)(p<0.01)$, and the changes were more stable from the postoperative period to the last follow-up $(p<0.01)$. Cage subsidence was observed significantly less in LLIF $(n=6)$ than TLIF $(n=21)$. Fusion rate was not different between the two groups. The clinical outcomes did not differ significantly at any time point between the two groups. Complications were not statistically significant. However, TLIF showed chronic mechanical problems with screw loosening in four patients and LLIF showed temporary symptoms associated with the surgical approach, such as psoas and ileus muscle symptoms in three and two cases, respectively.

Conclusion : LLIF was more effective than TLIF for spondylolisthesis reduction, likely due to the higher profile cage and ligamentotactic effect. In addition, LLIF showed mechanical stability of the reduction level by using a cage with a larger footprint. Therefore, LLIF should be considered a surgical option before TLIF for patients with unstable DS.

Key Words : Spinal fusion · Minimally invasive surgical procedures · Spondylolisthesis.

- Received : June 28, 2018 •Revised : July 20, 2018 •Accepted : September 19, 2018

- Address for reprints : Seung Won Park

Department of Neurosurgery, Chung-Ang University Hospital, 102 Heukseok-ro, Dongjak-gu, Seoul 06973, Korea

Tel : +82-2-6299-3190, Fax : +82-2-6299-2069, E-mail : nspsw@cau.ac.kr, ORCID : https://orcid.org/0000-0001-8305-7501

This is an Open Access article distributed under the terms of the Creative Commons Attribution Non-Commercial License (http://creativecommons.org/licenses/by-nc/4.0) which permits unrestricted non-commercial use, distribution, and reproduction in any medium, provided the original work is properly cited. 


\section{INTRODUCTION}

Degenerative spondylolisthesis (DS) is defined as the forward slippage of an upper vertebra with respect to a lower vertebra, which causes back pain or radiculopathy due to spinal canal stenosis or foraminal stenosis ${ }^{11)}$. DS occurs mainly in those above 50 years of age and about $73 \%$ of cases occur at the $\mathrm{L} 4-5$ level. The reported prevalence is $2.7 \%$ in men and $8.1 \%$ in women ${ }^{16)}$. In particular, the prevalence of spondylolisthesis in the Chinese population over 65 years is $19.1 \%$ in men and $25.0 \%$ in women, and spondylolisthesis progression and de novo spondylolisthesis occur in more than $12 \%$ of both men and women ${ }^{13,29)}$. A recent study on patients with symptomatic DS reported that patients who received surgery had significantly greater improvements in pain and function compared to those two received non-operative treatment in eight years of follow-up ${ }^{1)}$. Therefore, surgery is an important treatment in patients with symptomatic DS.

Among the surgical treatments for patients with symptomatic DS, intervertebral fusion is the main surgical method used to stabilize the spine and reduce pain and disability; its types include the posterolateral (posterior lumbar interbody fusion and transforaminal lumbar interbody fusion [TLIF]), lateral (lateral lumbar interbody fusion [LLIF], direct lateral interbody fusion [DLIF], extreme lateral interbody fusion [XLIF], and oblique lateral interbody fusion [OLIF]), and anterior (anterior lumbar interbody fusion) approaches ${ }^{9,18,24)}$. LLIF is a relatively new minimally invasive surgery (MIS) for the treatment of DS and has the advantage of being able to insert an interbody cage with a large foot-print without injury to posterior facets ${ }^{6}$. While recent findings on the treatment of DS with LLIF have been reported and comparative studies of LLIF with other therapies have been published, there remains controversy regarding whether LLIF is the optimal treatment for $\mathrm{DS}^{5,10,15,18,22,23,25,30)}$. In addition, direct comparison studies between TLIF and MIS-LLIF were not only small in number but also insufficient for explaining the recent advances of MIS-LLIF ${ }^{15,25)}$.

We compared the clinical and radiological outcomes of TLIF and MIS-LLIF (DLIF or OLIF), which have mainly performed for DS in our hospital, to investigate better fusion surgery for patients with symptomatic DS, whose numbers are increasing in aging societies.

\section{MATERIALS AND METHODS}

This study was a single-center study in which the surgeries were conducted by two neurosurgeons (Y.B.K. and S.W.P.). TLIF was performed only by Y.B.K. and LLIF was performed only by S.W.P. We retrospectively reviewed and enrolled patients with symptomatic DS who underwent TLIF (TLIF group) or MIS-LLIF (LLIF group) at the L4-5 level between January 2011 and December 2015 and were followed-up for more than one year. Age, sex, bone marrow density, and body mass index were evaluated. This study was reviewed and approved by the Institutional Review Board (CAUH IRB No. 1806-002-16175).

\section{Surgical techniques}

The TLIF group underwent unilateral open TLIF with a single capstone cage (Medtronic, Minneapolis, MN, USA) filled with autologous bone. The pedicle screw fixation (Zenius system, Medyssey, Jecheon, Korea) was done by a conventional open technique ipsilaterally and an interfascial approach contralaterally. All components for central stenosis and foraminal stenosis were decompressed directly during the TLIF approach.

The LLIF group underwent MIS-DLIF or OLIF by using a transpsoas or extrapsoas retroperitoneal approach and percutaneous pedicle screw fixation. The LLIF group was treated by using a Clydesdale cage (Medtronic, Memphis, TN, USA) filled with a demineralized bone matrix (DBM, Grafton; Medtronic, Minneapolis) and a percutaneous pedicle screw fixation system (Longitude II system, Medtronic, Memphis). All foraminal stenosis (23 of 39 patients) were decompressed indirectly by increasing the disc height and correcting the spondylolisthesis. Additional posterior decompression was performed for patients in the LLIF group (16 of 39 patients) with severe central stenosis.

\section{Clinical evaluation}

We assessed the clinical outcomes by using the visual analog scale (VAS) for back pain (VAS-back) and leg pain (VASleg), as well as the Oswestry disability index (ODI). VAS and ODI were evaluated preoperatively (PreVAS and PreODI) and one year postoperatively (PostVAS and PostODI). In addition, we also assessed postoperative complications, the length of hospital stay, and operation time. 


\section{Radiographic measurement}

We checked the spondylolisthesis rate, segmental sagittal angle (SSA), mean disc height (MDH), intervertebral foramen height (FH), and cage subsidence by simple radiographs. In addition, lumbar lordosis (LL) was measured by 36 -inch whole-spine X-ray films.

The spondylolisthesis rate (\%) was calculated by dividing the amount of L4 body displacement on the L5 upper endplate by the anterior-posterior diameter of the L5 upper endplate. The SSA was measured as the angle between the superior endplate of the L4 vertebra and the inferior endplate of the L5 vertebra. The MDH was calculated as the mean value of the anterior and posterior disc heights $(\mathrm{mm})$ in lateral images. The $\mathrm{FH}$ was measured as the distance between the inferior margin of the L4 pedicle and the superior margin of the L5 pedicle in the lateral view. The LL was measured as the angle between the superior endplates of L1 and S1. Cage subsidence was defined as when a cage sank into adjacent vertebral body more than 2 $\mathrm{mm}$ on the last follow-up (f/u) lateral image.

Fusion rate was evaluated by two methods. First, grade of 1 or 2 on plain radiography was defined as a successful bony fusion by using Bridwell-Lenke grading system (grade 1 : completely remodeled with trabeculae across the disc space; grade 2 : graft intact with no lucent lines between the graft and adjacent endplates; grade 3 : graft intact but with a radiolucent line between the graft and an adjacent endplate; and grade $4:$ lucency along the entire border of the graft or lucency around a pedicle screw or subsidence of the graft $)^{4)}$. Second, grade of 1 or 2 on computed tomography (CT) imaging at 1-year postoperative (1 YA) was defined as a successful bony fusion by using CT-based classification (grade I : complete fusion, implies cortical union of the allograft and central trabecular continuity; grade II : partial fusion, implies cortical union of the structural allograft with partial trabecular incorporation; grade III : unipolar pseudarthrosis, denotes superior or inferior cortical non-union of the central allograft with partial trabecular discontinuity centrally; and grade IV : bipolar pseudarthrosis, suggests both superior and inferior cortical nonunion with a complete lack of central trabecular continuity) ${ }^{27)}$.

The radiologic parameters were evaluated at preoperative (Preop), immediate postoperative (Postop), 1 YA, and last f/u. All radiologic measurements were made using the measuring tool within the picture archiving communication system in Chung-Ang Univertisy Hospital. Two observations were made at an interval of at least one week by two neurosurgeons (M.J.K. and S.W.P.), and the mean values were used for the study. The intraclass correlation coefficient (ICC) was checked to verify the intra-observer and inter-observer reliability of the radiologic measurements.

\section{Statistical analysis}

The radiological and clinical outcomes were compared in each group using paired t-tests. The outcomes between the DLIF and OLIF groups were compared using Student's t- and

Table 1. Baseline characteristics

\begin{tabular}{|c|c|c|c|}
\hline & TLIF group & LLIF group & $p$-value \\
\hline Number of patients & 41 & 39 & \\
\hline Sex ratio (male : female) & $9: 32$ & $11: 28$ & 0.518 \\
\hline Age (years) & $62.3 \pm 9.4$ & $59.6 \pm 9.3$ & 0.200 \\
\hline BMD (T-score) & $-1.0 \pm 1.2$ & $-1.2 \pm 1.4$ & 0.462 \\
\hline $\mathrm{BMI}\left(\mathrm{kg} / \mathrm{m}^{2}\right)$ & $25.1 \pm 4.5$ & $24.8 \pm 4.3$ & 0.770 \\
\hline Follow up period (months) & $27.2 \pm 18.0$ & $33.6 \pm 15.9$ & 0.096 \\
\hline Spondylolisthesis grade I & 34 & 28 & 0.289 \\
\hline Spondylolisthesis grade II & 7 & 11 & 0.289 \\
\hline \multicolumn{4}{|l|}{ Main pathology } \\
\hline Central canal stenosis & 19 & 13 & 0.261 \\
\hline Foraminal stenosis & 22 & 26 & 0.261 \\
\hline
\end{tabular}

Values are presented as mean \pm standard deviation or number unless otherwise indicated. TLIF : transforaminal lumbar interbody fusion, LLIF : lateral lumbar interbody fusion, BMD : bone marrow density, BMI : body mass index 
chi-square tests. $p$-values $<0.05$ were considered statistically significant. The ICC values were graded using previously described semiquantitative criteria (excellent for values in the $0.90-1.0$ range, good for $0.70-0.89$, fair/moderate for $0.50-$ 0.69 , low for $0.25-0.49$, and poor for $0.0-0.24$ ).

\section{RESULTS}

\section{Baseline characteristics}

There were 41 (male : female $=9: 32$ ) and 39 (male : female $=11$ : 28) patients in the groups, respectively. In these groups, 34 and 28 and seven and 11 patients had grade I and II spondylolisthesis, respectively. The mean follow-up period was $27.2 \pm 18.0$ and $33.6 \pm$ 15.9 months in the TLIF and LLIF groups, respectively. The baseline characteristics are described in Table 1, and there were no significant differences between the TLIF and LLIF groups.

\section{Clinical outcomes}

In the TLIF and LLIF groups, the PreVAS-back were 7.6 \pm 1.4 and $7.5 \pm 1.5$, the PreVAS-leg were $6.0 \pm 1.5$ and $6.6 \pm 2.0$, and the PreODI were $44.5 \pm 11.7$ and $45.6 \pm 12.6 \%$, respectively. The PostVAS-back were $2.2 \pm 1.3$ and $1.9 \pm 0.6$, the PostVAS-leg were $1.8 \pm 0.8$ and $1.6 \pm 0.7$, and the PostODI were 18.0 \pm 5.9 and $16.5 \pm$ $6.4 \%$, respectively. In both groups, both PostVAS and PostODI showed a significant improvement compared to the preoperative values $(p<0.001)$, and there were no significant differences between the two groups (Table 2).

Table 2. VAS and ODI in TLIF and LLIF groups

\begin{tabular}{lccc}
\hline & TLIF group & LLIF group & $p$-value \\
\hline PreVAS-back & $7.6 \pm 1.4$ & $7.5 \pm 1.5$ & 0.906 \\
PostVAS-back & $2.2 \pm 1.3^{*}$ & $1.9 \pm 0.6^{*}$ & 0.193 \\
PreVAS-leg & $6.0 \pm 1.5$ & $6.6 \pm 2.0$ & 0.147 \\
PostVAS-leg & $1.8 \pm 0.8^{*}$ & $1.6 \pm 0.7^{*}$ & 0.146 \\
PreODI (\%) & $44.5 \pm 11.7$ & $45.6 \pm 12.6$ & 0.686 \\
PostODI (\%) & $18.0 \pm 5.9^{*}$ & $16.5 \pm 6.4^{*}$ & 0.265 \\
\hline
\end{tabular}

Values are presented as mean \pm standard deviation. ${ }^{*} p<0.001$ compared to the preoperative value. VAS : visual analog scale, ODI : Oswestry disability index, TLIF : transforaminal lumbar interbody fusion, LLIF : lateral lumbar interbody fusion, Pre : preoperative, Post : postoperative 1 year

\section{Cage dimensions}

The cage height, length, width, and angle were $11.6 \pm 1.0 \mathrm{~mm}$, $31.1 \pm 1.0 \mathrm{~mm}, 10.0 \pm 0.0 \mathrm{~mm}$, and $0.0 \pm 0.0^{\circ}$ in the TLIF group, respectively, and $13.0 \pm 0.9 \mathrm{~mm}, 47.8 \pm 3.0 \mathrm{~mm}, 21.3 \pm 1.6 \mathrm{~mm}$, and $10.6 \pm 2.6^{\circ}$ in the LLIF group, respectively. The cages in the LLIF group were significantly taller, longer, wider, and had larger angles than those in the TLIF group $(p<0.001)$ (Table 3$)$.

\section{Perioperative parameters}

The mean hospital stays were $6.9 \pm 1.4$ and $7.1 \pm 1.9$ days in the TLIF and LLIF groups, respectively and there was no significant difference. The mean operation times were $127.4 \pm 21.8$ and 116.4 \pm 23.8 minutes in the TLIF and LLIF groups, respectively, and the LLIF group had a significantly shorter operation time than that in the TLIF group ( $p=0.034)$.

The complications observed during follow-up included four instances of screws loosening (one reoperation) in the TLIF group and three psoas muscle symptoms and two postoperative ileus in the LLIF group. In the LLIF group, there were no instances of genitofemoral nerve symptoms, sympathetic symptom, or screw loosening and all complications improved within a few days. There was no surgical site infection or fatal complications in either group. The types of complications differed between groups, but the difference was not statistically significant (Table 3).

Table 3. Perioperative parameters and cage dimension in TLIF and LLIF

\begin{tabular}{lcc}
\hline & TLIF group & LLIF group \\
\hline Length of hospital stay (day) & $6.9 \pm 1.4$ & $7.1 \pm 1.9$ \\
Operation time (minutes) & $127.4 \pm 21.8$ & $116.4 \pm 23.8^{*}$ \\
Complication & $4(9.8)$ & $5(12.8)$ \\
Psoas muscle symptoms & 0 & $3(5.1)$ \\
lleus & 0 & $2(5.1)$ \\
Screw loosening (number) & $4(9.8)$ & 0 \\
Cage dimension & & $13.0 \pm 0.9^{\dagger}$ \\
Height (mm) & $11.6 \pm 1.0$ & $47.8 \pm 3.0^{\dagger}$ \\
Length (mm) & $31.1 \pm 1.0$ & $21.3 \pm 1.6^{\dagger}$ \\
Width (mm) & $10.0 \pm 0.0$ & $10.6 \pm 2.6^{\dagger}$ \\
Lordotic angle $\left(^{\circ}\right.$ ) & $0.0 \pm 0.0$ & \\
\hline
\end{tabular}

Values are presented as mean \pm standard deviation or number (\%). ${ }^{*} p<0.05 .{ }^{\dagger} p<0.001$ compared to TLIF group. TLIF : transforaminal lumbar interbody fusion, LLIF : lateral lumbar interbody fusion 


\section{Radiographic parameters (Table 4)}

There were no significant differences in Preop-spondylolisthesis rate, MDH, FH, SSA, and LL between the TLIF and LLIF groups.

Table 4. Comparison of radiologic measurements between TLIF and LLIF

\begin{tabular}{|c|c|c|}
\hline & TLIF group $(n=41)$ & LLIF group $(n=39)$ \\
\hline \multicolumn{3}{|c|}{ Spondy rate (\%) } \\
\hline Preop & $18.2 \pm 6.7$ & $20.0 \pm 7.7$ \\
\hline Postop & $7.2 \pm 6.1$ & $3.8 \pm 3.8^{*}$ \\
\hline $1 Y A$ & $8.1 \pm 6.1$ & $4.1 \pm 3.9^{*}$ \\
\hline Last f/u & $10.1 \pm 6.6$ & $4.6 \pm 9.3^{\dagger}$ \\
\hline \multicolumn{3}{|l|}{$\mathrm{MDH}(\mathrm{mm})$} \\
\hline Preop & $7.6 \pm 2.1$ & $8.5 \pm 3.0$ \\
\hline Postop & $10.3 \pm 1.5$ & $13.9 \pm 1.4^{\dagger}$ \\
\hline $1 Y A$ & $8.3 \pm 1.3$ & $13.3 \pm 1.5^{\dagger}$ \\
\hline Last f/u & $7.7 \pm 1.4$ & $12.6 \pm 1.8^{\dagger}$ \\
\hline \multicolumn{3}{|l|}{$\mathrm{FH}(\mathrm{mm})$} \\
\hline Preop & $16.8 \pm 2.3$ & $17.3 \pm 2.7$ \\
\hline Postop & $19.4 \pm 1.7$ & $21.9 \pm 1.9^{\dagger}$ \\
\hline $1 Y A$ & $17.2 \pm 1.8$ & $21.5 \pm 2.0^{\dagger}$ \\
\hline Last f/u & $16.8 \pm 1.8$ & $20.7 \pm 2.1^{\dagger}$ \\
\hline \multicolumn{3}{|l|}{ SSA $\left({ }^{\circ}\right)$} \\
\hline Preop & $13.7 \pm 6.9$ & $13.0 \pm 8.2$ \\
\hline Postop & $15.6 \pm 5.2$ & $18.9 \pm 5.3^{*}$ \\
\hline $1 Y A$ & $13.5 \pm 5.8$ & $18.6 \pm 5.4^{\dagger}$ \\
\hline Last f/u & $13.3 \pm 6.0$ & $18.3 \pm 5.5^{\dagger}$ \\
\hline \multicolumn{3}{|l|}{$\operatorname{LL}\left({ }^{\circ}\right)$} \\
\hline Preop & $42.1 \pm 9.5$ & $43.3 \pm 11.5$ \\
\hline Postop & $43.3 \pm 8.6$ & $46.1 \pm 9.3$ \\
\hline $1 Y A$ & $43.2 \pm 9.4$ & $46.5 \pm 9.5$ \\
\hline Last f/u & $43.8 \pm 9.8$ & $46.4 \pm 10.2$ \\
\hline \multicolumn{3}{|c|}{ Fusion on CT } \\
\hline $1 Y A$ & $38(92.7)$ & $36(92.3)$ \\
\hline \multicolumn{3}{|c|}{ Fusion on X-ray } \\
\hline $6 \mathrm{MA}$ & $36(87.8)$ & $35(85.4)$ \\
\hline $1 Y A$ & $38(92.7)$ & 37 (94.8) \\
\hline Last f/u & $38(92.7)$ & $39(100.0)$ \\
\hline \multicolumn{3}{|c|}{ Cage subsidence } \\
\hline Last f/u & 21 & $6^{\dagger}$ \\
\hline
\end{tabular}

Values are presented as mean \pm standard deviation or number (\%). ${ }^{*} p<0.01$. ${ }^{\dagger} p<0.001$ compared to TLIF group. TLIF : transforaminal lumbar interbody fusion, LLIF : lateral lumbar interbody fusion, Spondy : spondylolisthesis, Preop : preoperative, Postop : immediate postoperative, 1 YA : postoperative 1 year, f/u : follow-up, MDH : mean disc height, $\mathrm{FH}$ : intervertebral foramen height, SSA : segmental sagittal angle, LL : lumbar lordosis, CT : computed tomography, 6 MA : postoperative 6 months
The spondylolisthesis rate of Postop, $1 \mathrm{YA}$, and last f/u were 7.2 $\pm 6.1,8.1 \pm 6.1$, and $10.1 \pm 6.6 \%$ in the TLIF group, respectively, and $3.8 \pm 3.8$ ( $p=0.005), 4.1 \pm 3.9(p=0.001)$, and $4.6 \pm 9.3 \%(p<0.001)$ in the LLIF group, respectively. The spondylolisthesis rates after surgery were significantly lower in the LLIF group than those in the TLIF group at all postoperative periods.

The MDH of Postop, $1 \mathrm{YA}$, and last $\mathrm{f} / \mathrm{u}$ were $10.3 \pm 1.5,8.3 \pm 1.3$, and $7.7 \pm 1.4 \mathrm{~mm}$ in the TLIF group, respectively, and $13.9 \pm 1.4$, $13.3 \pm 1.5$, and $12.6 \pm 1.8 \mathrm{~mm}$ in the LLIF group, respectively. The MDH's were significantly higher in the LLIF group than those in the TLIF group at all postoperative periods $(p<0.001)$.

The FH of Postop, 1 YA, and last $\mathrm{f} / \mathrm{u}$ were 19.4 $\pm 1.7,17.2 \pm 1.8$, and $16.8 \pm 1.8 \mathrm{~mm}$ in the TLIF group, respectively, and $21.9 \pm$ $1.9,21.5 \pm 2.0$, and $20.7 \pm 2.1 \mathrm{~mm}$ in the LLIF group, respectively. The FH's were significantly higher in the LLIF group than those in the TLIF group at all postoperative periods $(p<0.001)$.

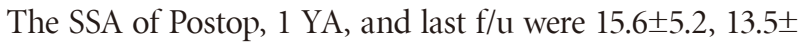
5.8 , and $13.3 \pm 6.0^{\circ}$ in the TLIF group, respectively, and $18.9 \pm 5.3$ ( $p=0.007), 18.6 \pm 5.4(p<0.001)$, and $18.3 \pm 5.5^{\circ}(p<0.001)$ in the LLIF group, respectively. The SSA's were significantly larger in the LLIF group than those in the TLIF group at all postoperative periods.

The LL of Postop, 1 YA, and last f/u were 43.3 $\pm 8.6,43.2 \pm 9.4$, and $43.8 \pm 9.8^{\circ}$ in the TLIF group, respectively, and $46.1 \pm 9.3$, $46.1 \pm 9.5$, and $46.4 \pm 10.2^{\circ}$ in the LLIF group, respectively. There were no significant differences between the two groups.

The number of cage subsidence was 21 and six in the TLIF and LLIF groups, respectively, and cage subsidence was significantly less in LLIF group $(p<0.001)$.

In the TLIF group, the mean difference between Postop and last $\mathrm{f} / \mathrm{u}$ values for the spondylolisthesis rate, $\mathrm{MDH}, \mathrm{FH}$, and SSA were $-2.9 \pm 3.9 \%$ (-63.8\%), $2.6 \pm 1.3 \mathrm{~mm}(24.7 \%), 2.5 \pm 1.4$

Table 5. Mean changes $(\Delta)$ of radiologic parameters between the immediate postoperative and the last follow-up values

\begin{tabular}{lcc}
\hline & TLIF group & LLIF group \\
\hline$\triangle$ Spondy rate (\%) & $-63.8 \pm 105.2$ & $-16.2 \pm 34.7^{*}$ \\
$\triangle \mathrm{MDH}(\%)$ & $24.7 \pm 11.3$ & $9.7 \pm 7.6^{\dagger}$ \\
$\triangle \mathrm{FH}(\%)$ & $13.2 \pm 6.9$ & $5.5 \pm 4.2^{\dagger}$ \\
$\triangle \mathrm{SSA}(\%)$ & $17.4 \pm 19.4$ & $2.9 \pm 9.3^{\dagger}$ \\
\hline
\end{tabular}

Values are presented as mean \pm standard deviation. ${ }^{*} p<0.01 .{ }^{\dagger} p<0.001$ compared to TLIF group. TLIF : transforaminal lumbar interbody fusion, LLIF : lateral lumbar interbody fusion, Spondy : spondylolisthesis, MDH : mean disc height, FH : intervertebral foramen height, SSA : segmental sagittal angle 
$\mathrm{mm}(13.2 \%)$, and $2.3 \pm 2.5^{\circ}(17.4 \%)$, respectively. The corresponding values in the LLIF group were $-0.8 \pm 1.5 \%$ (-16.2\%), $1.3 \pm 1.0 \mathrm{~mm}(9.7 \%), 1.2 \pm 0.9 \mathrm{~mm}(5.5 \%)$, and $-0.5 \pm 1.5^{\circ}(2.9 \%)$, respectively, and the differences were significantly less than those in the TLIF group (Table 5).

The fusion rate on plain radiography was $87.8 \%$ and $85.4 \%$ at postoperative 6 months, $92.7 \%$ and $94.8 \%$ at $1 \mathrm{YA}$, and 92.7\% and $100.0 \%$ at last $\mathrm{f} / \mathrm{u}$ in TLIF and LLIF groups, respectively. The fusion rate on CT scan was $92.7 \%$ and $92.3 \%$ at $1 \mathrm{YA}$ in the TLIF and LLIF groups, respectively, and there was no significant difference between the two groups on plain radiography and CT.

The intra- and inter-observer ICCs were $0.85-0.94$ and $0.75-0.92$, respectively (Table 6).

Table 6. Strength of agreement of inter- and intra-observer analysis for parameters

\begin{tabular}{lcc}
\hline & Intra-observer ICC Inter-observer ICC \\
\hline Spondylolisthesis rate & 0.93 & 0.90 \\
MDH & 0.88 & 0.78 \\
FH & 0.86 & 0.80 \\
SSA & 0.89 & 0.83 \\
LL & 0.94 & 0.92 \\
Cage subsidence & 0.92 & 0.89 \\
Fusion rate & 0.85 & 0.75 \\
\hline
\end{tabular}

ICC : intraclass correlation coefficient, $\mathrm{MDH}$ : mean disc height, $\mathrm{FH}$ : intervertebral foramen height, SSA : segmental sagittal angle, LL : lumbar lordosis

\section{DISCUSSION}

TLIF and LLIF use different mechanisms to decompress stenosis due to DS (direct vs. indirect decompression). TLIF provides a good visualization of the neural elements through direct decompression of the posterior element ${ }^{8,12)}$. LLIF approaches the intervertebral disc space through retroperitoneal transpsoas (DLIF/XLIF) or extrapsoas (OLIF) and indirectly decompresses the spinal canal and intervertebral foramen by inserting a cage with large footprint ${ }^{5,10,23)}$.

Sembrano et al. ${ }^{25)}$ reported the clinical outcomes of TLIF and XLIF in patients with DS. They reported high mean improvements in pain $(73 \%$ and $64 \%$ in back pain, $79 \%$ and $74 \%$ in leg pain), disability (53\% and 57\%), and quality of life (63\% and 64\%) in both the TLIF and XLIF groups, with no significant differences between the two groups. In our study, PostVAS-back and PostVAS-leg were improved by $71.1 \%$ and $70 \%$ in the TLIF group, respectively, and by $74.7 \%$ and $75.8 \%$ in the LLIF group, respectively. In addition, PostODI was improved by $59.6 \%$ and $63.6 \%$ in the TLIF and LLIF groups, respectively. According to our results, TLIF and LLIF significantly improved pain and disability in patients with DS without significant differences between the two groups, similar to the results of previous studies ${ }^{6,25}$.

A prospective study of radiological outcomes of TLIF and XLIF compared different mechanisms (direct vs. indirect), reporting that the average postoperative disc height was significantly higher in the XLIF group $(10.1 \pm 2.1 \mathrm{~mm})$ than that in
(A)

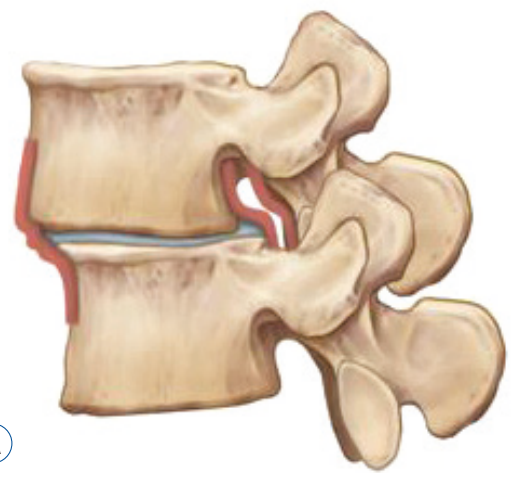

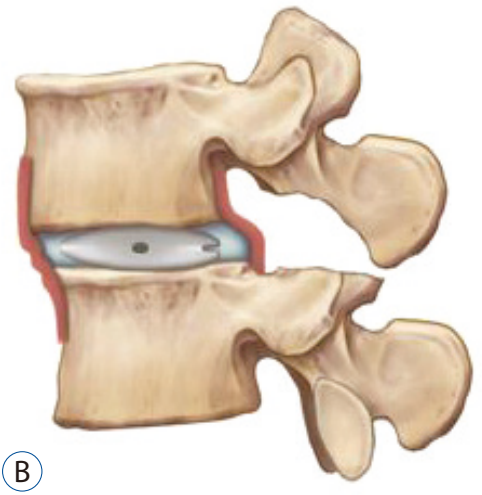

(B)

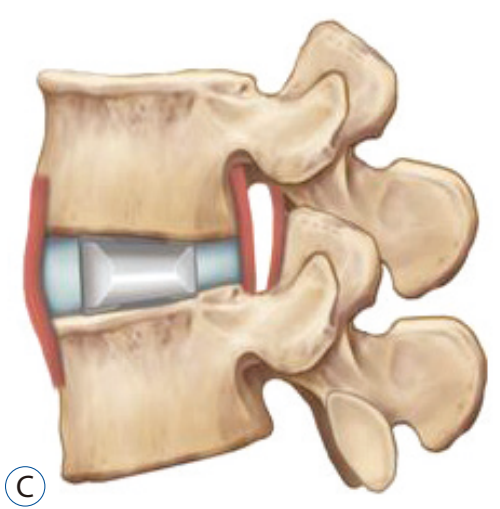

Fig. 1. Spondylolisthesis reduction in TLIF and LLIF. In preoperative state of DS, disc bulging and bucking of posterior longitudinal ligament and ligamentum flavum cause stenosis (A). TLIF improves stenosis through direct decompression of posterior elements, but it is difficult to insert of a cage with sufficient height, which is disadvantageous in terms of the spondylolisthesis reduction (B). LLIF can maximize the ligamentotaxic effect through insertion of cage with sufficient height, which indirectly decompresses the foraminal stenosis as well as corrects the DS effectively (C). TLIF : transforaminal lumbar interbody fusion, LLIF : lateral lumbar interbody fusion, DS : degenerative spondylolisthesis. 

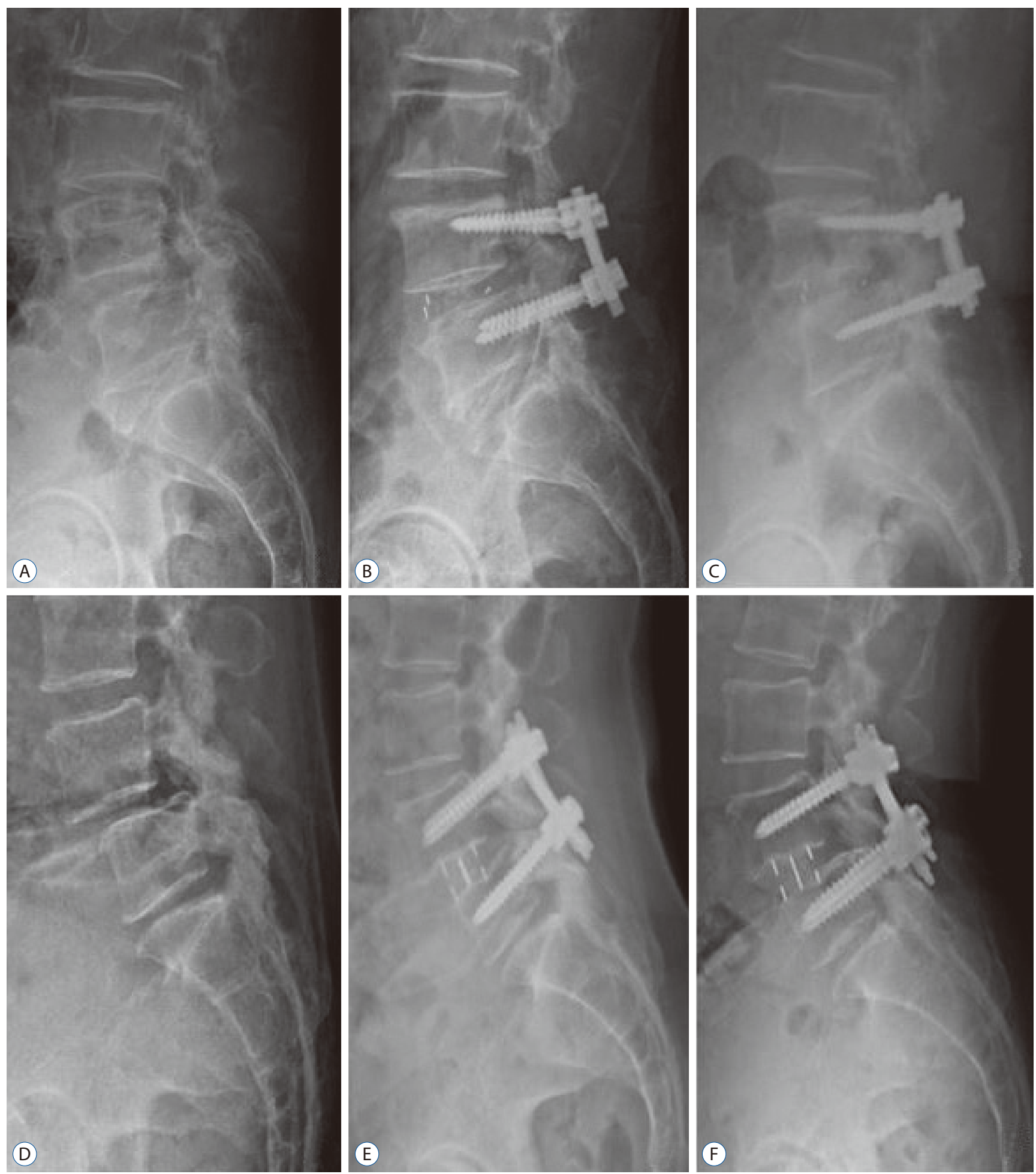

Fig. 2. Cases showing the largest change of reduced spondylolisthesis from immediate postoperative to last follow-up X-ray. In TLIF case, spondylolisthesis rate was $23.6 \%$ at preoperative (A), and reduced to $7.5 \%$ at immediately postoperative (B). It was slipped to $21.2 \%$ at 23 months after surgery (C). In LLIF case, spondylolisthesis rate was $28.7 \%$ at preoperative (D), and reduced to $6.7 \%$ at immediately postoperative (E). Then, it was $10.4 \%$ at 45 months after surgery (F). TLIF : transforaminal lumbar interbody fusion, LLIF : lateral lumbar interbody fusion. 
the TLIF group $(9.1 \pm 1.8 \mathrm{~mm})$ and that the foraminal dimension was significantly larger in the XLIF group than that in the TLIF group ${ }^{15)}$. In our study, both Postop-MDH and PostopFH were significantly higher in the LLIF group $(13.9 \pm 1.4 \mathrm{~mm}$ and $21.9 \pm 1.9 \mathrm{~mm}$, respectively) than those in the TLIF group (10.3 $\pm 1.5 \mathrm{~mm}$ and $19.4 \pm 1.7 \mathrm{~mm}$, respectively); these results are similar to those of other studies ${ }^{7,19,26)}$. These results indicate that LLIF is effective for indirect decompression by increasing disc height more than that with TLIF.

However, unlike a previous study ${ }^{15)}$, the Postop-MDH difference between the TLIF and LLIF groups was larger and the

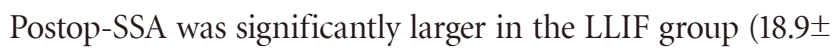
$\left.5.3^{\circ}\right)$ than that in the TLIF group $\left(15.6 \pm 5.2^{\circ}\right)$ in our study. The differences between the two studies are likely due to differences in cage dimension, which is similar to the results of other studies $^{2,26)}$. In a previous study ${ }^{15)}$, the mean cage heights were 10.6 and $11.2 \mathrm{~mm}$ and the mean cage angles were $0^{\circ}$ and $5.4^{\circ}$ in the TLIF and XLIF groups, respectively. However, in our study, the mean cage heights were $11.6 \pm 1.0$ and $13.0 \pm 0.9 \mathrm{~mm}$ and the mean cage angles were $0.0 \pm 0.0$ and $10.6 \pm 2.6^{\circ}$ in the TLIF and LLIF groups, respectively.

Therefore, LLIF has the advantage that the operator can adjust the SSA by selecting the cage angle. In addition, LLIF can improve the radicular symptoms through indirect decompression by increasing the $\mathrm{MDH}$ and $\mathrm{FH}$ via the insertion of a cage with a higher profile than that in TLIF.

The superiority of LLIF for spondylolisthesis reduction has been reported in several studies, ${ }^{5,10,15)}$, and LLIF was also reported to be effective in the reduction of even grade II spondylolisthesis $^{30)}$. However, a previous study comparing it to TLIF reported that LLIF had no advantage in terms of spondylolisthesis reduction $^{15)}$. In our study, the Postop-spondylo-

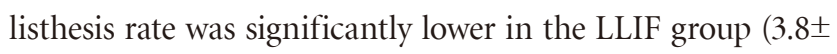
$3.8 \%)$ than that in the TLIF group $(7.2 \pm 6.1 \%)$; therefore, LLIF resulted in a significant correction. The difference observed between the two studies may be due to differences in ligamentotactic effects. The ligamentotactic effect is a method used for the indirect reduction of a fracture by the application of a strong distraction force that is transmitted through intact ligaments and capsule ${ }^{17)}$. It is used in degenerative disease as well as fracture ${ }^{21)}$. During cage insertion, the cage height acts as a distraction force. The cage height used in our study (average, $13.0 \mathrm{~mm}$ ) was higher than that used in the previous study (average, $11.2 \mathrm{~mm}$ ); thus, a sufficient distraction force could be created for spondylolisthesis reduction via the ligamentotactic effect (Fig. 1).

A number of studies have reported that one-level fusion can also affect the overall $\mathrm{LL}^{2,3,14,20,28)}$, however, in our study, onelevel fusion did not seem to have a significant effect on LL even though there was a significant difference in SSA between the TLIF and LLIF groups.

Finally, in our study, the changes in the spondylolisthesis rate, $\mathrm{MDH}, \mathrm{FH}$, and SSA between Postop and last f/u were significantly smaller in the LLIF group than those in the TLIF group, a finding similar to that of a previous study, suggesting that LLIF is stable over the long term ${ }^{15)}$ (Fig. 2). The cage used in LLIF was sufficiently larger and longer to contact the epiphyseal ring at both sides than the cage used in TLIF, which may have caused the changes. The difference in cage subsidence could also be due to this reason. In our study, although there was no difference in complications between the TLIF and LLIF groups, the complications in the LLIF group included symptoms associated with the surgical approach, whereas complications in the TLIF group included mechanical failure due to screw loosening. Although the number was small, screw loosening was present only in the TLIF group, which is indirect evidence of the excellent stability of LLIF. In four patients with screw loosening, the PostVAS-back, PostVAS-leg, and PostODI were $5.5 \pm 1.3,3.5 \pm 0.8,26.3 \pm 3.5 \%$, respectively, which was not good. Although a significant difference in the clinical outcome was not observed due to the small numbers, a more accurate assessment will be possible in large-scale studies with longer follow-up periods.

The present retrospective study has several limitations. The two types of operations were performed by different two surgeons at a single hospital. Although baseline characteristics of the patients were matched, surgeon bias could not be eliminated because the treatment choice was not randomized. We did not elucidate the difference between DLIF and OLIF, which were mixed in the LLIF group. Although significant differences were observed in the parameters for long-term mechanical stability, bias was present due to the different types of pedicle screw systems between the groups. Finally, our study was relatively small and had a short follow-up period. A larger-prospective multicenter study with a longer follow-up period is necessary to provide additional data on the optimal treatment for DS. 


\section{CONCLUSION}

The number of patients with DS is increasing with the aging society and the need for fusion surgery in patients with symptomatic DS is also increasing. According to the results of our study, both TLIF and LLIF showed good clinical and radiological results in patients with symptomatic DS. However, the LLIF was significantly superior to the TLIF in spondylolisthesis reduction, $\mathrm{MDH}$ and $\mathrm{FH}$ increase, and SSA correction as a result of the taller and larger angled cage and ligamentotactic effect. In addition, the LLIF showed mechanical stability of the reduction level due to the use of a cage with a greater footprint. Therefore, LLIF should be considered a surgical option before TLIF for patients with unstable DS requiring significant reduction.

\section{CONFLICTS OF INTEREST}

No potential conflict of interest relevant to this article was reported.

\section{INFORMED CONSENT}

Informed consent was obtained from all individual participants included in this study.

\section{AUTHOR CONTRIBUTIONS}

\author{
Conceptualization : MJK, SWP \\ Data curation : MJK \\ Formal analysis : MJK \\ Methodology : SWP, YBK \\ Project administration : SWP \\ Visualization : MJK \\ Writing - original draft : MJK \\ Writing - review \& editing : MJK, SWP, YBK
}

\section{References}

1. Abdu WA, Sacks OA, Tosteson ANA, Zhao W, Tosteson TD, Morgan TS, et al. : Long-term results of surgery compared with nonoperative treatment for lumbar degenerative spondylolisthesis in the spine patient outcomes research trial (SPORT). Spine (Phila Pa 1976) 43 : 1619-1630, 2018

2. Ahlquist S, Park HY, Gatto J, Shamie AN, Park DY : Does approach matter? A comparative radiographic analysis of spinopelvic parameters in single-level lumbar fusion. Spine J 18 : 1999-2008, 2018

3. Anand N, Cohen RB, Cohen J, Kahndehroo B, Kahwaty S, Baron E: the influence of lordotic cages on creating sagittal balance in the CMIS treatment of adult spinal deformity. Int J Spine Surg $11: 23,2017$

4. Bridwell KH, Lenke LG, McEnery KW, Baldus C, Blanke K : Anterior fresh frozen structural allografts in the thoracic and lumbar spine. Do they work if combined with posterior fusion and instrumentation in adult patients with kyphosis or anterior column defects? Spine (Phila Pa 1976) 20 : 1410-1418, 1995

5. Campbell PG, Nunley PD, Cavanaugh D, Kerr E, Utter PA, Frank K, et al. : Short-term outcomes of lateral lumbar interbody fusion without decompression for the treatment of symptomatic degenerative spondylolisthesis at L4-5. Neurosurg Focus 44 : E6, 2018

6. Derman PB, Albert TJ : Interbody fusion techniques in the surgical management of degenerative lumbar spondylolisthesis. Curr Rev Musculoskelet Med $10:$ 530-538, 2017

7. Elowitz EH : Central and foraminal indirect decompression in MIS lateral interbody fusion (XLIF): video lecture. Eur Spine J 24 Suppl 3 : 449450, 2015

8. Foley KT, Holly LT, Schwender JD : Minimally invasive lumbar fusion. Spine (Phila Pa 1976) 28(15 Suppl) : S26-S35, 2003

9. Ghogawala Z, Dziura J, Butler WE, Dai F, Terrin N, Magge SN, et al. : Laminectomy plus fusion versus laminectomy alone for lumbar spondyIolisthesis. N Engl J Med 374 : 1424-1434, 2016

10. Goyal A, Kerezoudis P, Alvi MA, Goncalves S, Bydon M : Outcomes following minimally invasive lateral transpsoas interbody fusion for degenerative low grade lumbar spondylolisthesis: a systematic review. Clin Neurol Neurosurg 167 : 122-128, 2018

11. Guigui P, Ferrero E : Surgical treatment of degenerative spondylolisthesis. Orthop Traumatol Surg Res 103(1S) : S11-S20, 2017

12. Harms J, Rolinger $\mathrm{H}:$ A one-stager procedure in operative treatment of spondylolistheses: dorsal traction-reposition and anterior fusion (author's transl). Z Orthop Ihre Grenzgeb 120 : 343-347, 1982

13. He LC, Wang YX, Gong JS, Griffith JF, Zeng XJ, Kwok AW, et al. : Prevalence and risk factors of lumbar spondylolisthesis in elderly Chinese men and women. Eur Radiol 24 : 441-448, 2014

14. Hong TH, Cho KJ, Kim YT, Park JW, Seo BH, Kim NC : Does lordotic angle of cage determine lumbar lordosis in lumbar interbody fusion? Spine (Phila Pa 1976) 42 : E775-E780, 2017

15. Isaacs RE, Sembrano JN, Tohmeh AG; SOLAS Degenerative Study Group : Two-year comparative outcomes of MIS lateral and MIS transforaminal interbody fusion in the treatment of degenerative spondylolisthesis: Part II: radiographic findings. Spine (Phila Pa 1976) 41 Suppl 8 : S133S144, 2016

16. Jacobsen S, Sonne-Holm S, Rovsing H, Monrad H, Gebuhr P : Degen- 
erative lumbar spondylolisthesis: an epidemiological perspective: the Copenhagen osteoarthritis study. Spine (Phila Pa 1976) 32 : 120-125, 2007

17. Kuner EH, Kuner A, Schlickewei W, Mullaji AB : Ligamentotaxis with an internal spinal fixator for thoracolumbar fractures. J Bone Joint Surg Br 76 : 107-112, 1994

18. Lee CW, Yoon KJ, Ha SS : Which approach is advantageous to preventing development of adjacent segment disease? Comparative analysis of 3 different lumbar interbody fusion techniques (ALIF, LLIF, and PLIF) in L4-5 spondylolisthesis. World Neurosurg 105 : 612-622, 2017

19. Lee YS, Kim YB, Park SW, Chung C : Comparison of transforaminal lumbar interbody fusion with direct lumbar interbody fusion: clinical and radiological results. J Korean Neurosurg Soc 56 : 469-474, 2014

20. Melikian R, Yoon ST, Kim JY, Park KY, Yoon C, Hutton W : Sagittal plane correction using the lateral transpsoas approach: a biomechanical study on the effect of cage angle and surgical technique on segmental lordosis. Spine (Phila Pa 1976) 41 : E1016-E1021, 2016

21. Min JH, Jang JS, Kim SK, Maeng DH, Lee SH : The ligamentotactic effect on a herniated disc at the level adjacent to the anterior lumbar interbody fusion : report of two cases. J Korean Neurosurg Soc 46 : 65-67, 2009

22. Pawar AY, Hughes AP, Sama AA, Girardi FP, Lebl DR, Cammisa FP : A comparative study of lateral lumbar interbody fusion and posterior lumbar interbody fusion in degenerative lumbar spondylolisthesis. Asian Spine J 9 : 668-674, 2015

23. Pereira EA, Farwana M, Lam KS : Extreme lateral interbody fusion relieves symptoms of spinal stenosis and low-grade spondylolisthesis by indirect decompression in complex patients. J Clin Neurosci 35 : 5661, 2017
24. Resnick DK, Watters WC 3rd, Sharan A, Mummaneni PV, Dailey AT, Wang JC, et al. : Guideline update for the performance of fusion procedures for degenerative disease of the lumbar spine. Part 9: lumbar fusion for stenosis with spondylolisthesis. J Neurosurg Spine 21 : 5461, 2014

25. Sembrano JN, Tohmeh A, Isaacs R; SOLAS Degenerative Study Group : Two-year comparative outcomes of MIS lateral and MIS transforaminal interbody fusion in the treatment of degenerative spondylolisthesis: Part I: clinical findings. Spine (Phila Pa 1976) 41 Suppl 8 : S123-S132, 2016

26. Sembrano JN, Yson SC, Horazdovsky RD, Santos ER, Polly DW Jr : Radiographic comparison of lateral lumbar interbody fusion versus traditional fusion approaches: analysis of sagittal contour change. Int J Spine Surg $9: 16,2015$

27. Tan GH, Goss BG, Thorpe PJ, Williams RP : CT-based classification of long spinal allograft fusion. Eur Spine J 16 : 1875-1881, 2007

28. Uribe JS, Harris JE, Beckman JM, Turner AW, Mundis GM, Akbarnia BA : Finite element analysis of lordosis restoration with anterior longitudinal ligament release and lateral hyperlordotic cage placement. Eur Spine J 24 Suppl $3:$ 420-426, 2015

29. Wáng $Y X$, Deng $M$, Griffith JF, Kwok AW, Leung JC, Ahuja AT, et al. : Lumbar spondylolisthesis progression and de novo spondylolisthesis in elderly Chinese men and women: a year-4 follow-up study. Spine (Phila Pa 1976) 41 : 1096-1103, 2016

30. Xu DS, Bach K, Uribe JS : Minimally invasive anterior and lateral transpsoas approaches for closed reduction of grade II spondylolisthesis: initial clinical and radiographic experience. Neurosurg Focus 44 : E4, 2018 